\title{
CARACTERÍSTICA DA DEPOSIÇÃO E DISTRIBUIÇÃO DA CALDA DE PULVERIZAÇÃO NA CULTURA DA SOJA EM ESTÁDIO FENOLÓGICO V 6
}

\author{
JAMIL CONSTANTIN ${ }^{1}$, JOSÉ G. C. SALES ${ }^{1}$, CLEBER D. DE G. MACIEL ${ }^{1}$
}

RESUMO: O trabalho teve como objetivo avaliar a eficácia da deposição da calda de pulverização produzida por diferentes modelos de pontas de pulverização e pressões de trabalho no estádio fenológico $\mathrm{V}_{6}$ da cultura da soja. Oito tratamentos e cinquenta repetições foram estudados em delineamento inteiramente casualizado, em esquema fatorial $4 \times 2$, representados por quatro modelos de pontas de pulverização do fabricante Magno ${ }^{\circledR}$ e duas condições de pressão de trabalho (207 e $414 \mathrm{kPa}$ ), constituindo os tratamentos: AD 11002 (152 $\mathrm{L} \mathrm{ha}^{-1}$ e $\left.208 \mathrm{~L} \mathrm{ha}^{-1}\right)$; AD/D 11002 (152 L ha'-1 e $208 \mathrm{~L} \mathrm{ha}^{-1}$ ); AD-IA/D 11002 (152 L ha-1 e $208 \mathrm{~L} \mathrm{ha}^{-1}$ ); MAG 2 e MAG 3 (157 L ha ${ }^{-1}$ e $\left.212 \mathrm{~L} \mathrm{ha}^{-1}\right)$. Para monitorar a deposição das caldas de pulverização, utilizou-se dos traçadores Azul Brilhante FD\&C-1 (0,3\% p/v) e Amarelo de Tartrasina FD\&C-5 (0,6\% p/v). Os depósitos unitários das soluções sobre os trifólios superiores e inferiores das plantas de soja foram quantificados por espectrofotometria. As maiores quantidades de deposição da calda de pulverização, nas posições superior e inferior da cultura da soja, foram obtidas com as pontas MAG 2 e AD/D 11002, em pressão de $414 \mathrm{kPa}$. O aumento da pressão de 207 para $414 \mathrm{kPa}$, utilizando-se das pontas $\mathrm{AD}$ 11002; AD/D 11002 e AD-IA/D 11002 aumentou a deposição sobre os trifólios superiores e inferiores, ao contrário do uso da ponta MAG 3 em relação a MAG 2, em 414 kPa.

PALAVRAS-CHAVE: tecnologia de aplicação, corante, pontas de pulverização, Glycine max.

\section{CHARACTERISTIC OF THE SPRAY DEPOSITION AND DISTRIBUTION IN SOYBEAN'CROP ON V 6 GROWTH STAGE}

\begin{abstract}
This work aimed to evaluate the performance of spray deposition produced by different spray nozzles and work pressures in soybean crop on $\mathrm{V}_{6}$ phenological stage. Eight treatments and fifty repetitions were studied in completely randomized delineation, in factorial scheme $4 \times 2$, represented by four models of spray nozzles of Magno ${ }^{\mathrm{TM}}$ manufacturer and by two work pressures (207 and $414 \mathrm{kPa})$, constituting the treatments AD $11002\left(152 \mathrm{~L} \mathrm{ha}^{-1}\right.$ and $\left.208 \mathrm{~L} \mathrm{ha}^{-1}\right)$, AD/D 11002 (152 L ha-1 and $\left.208 \mathrm{~L} \mathrm{ha}^{-1}\right)$, AD-IA/D $11002\left(152 \mathrm{~L} \mathrm{ha}^{-1}\right.$ and $\left.208 \mathrm{~L} \mathrm{ha}^{-1}\right)$, MAG 2 and MAG 3 (157 L ha ${ }^{-1}$ and $\left.212 \mathrm{~L} \mathrm{ha}^{-1}\right)$. Bright Blue FD\&C-1 (0.3\% p/v) and Tartrazine Yellow FD\&C-5 $(0.6 \% \mathrm{p} / \mathrm{v})$ were used as tracers to monitoring the spray deposition. The unitary deposits of solutions over the upper and lower trifoliates from the soybean plants were quantified by spectrophotometer. The greatest amounts of spray deposition, at upper and lower positions of soybean crop, were obtained with MAG 2 and AD/D 11002 nozzles, with $414 \mathrm{kPa}$ pressure. The pressure increase from 207 to $414 \mathrm{kPa}$ using AD11002, AD/D 11002 and AD-IA/D 11002 nozzles raised the deposition over upper and lower trifoliates, in opposition to the use of MAG 3 nozzle in relation to MAG 2, in $414 \mathrm{kPa}$.
\end{abstract}

KEYWORDS: application technology, tracer, spray nozzles, Glycine max.

\section{INTRODUÇÃO}

A sojicultura vivencia uma busca incansável por altas produtividades agrícolas, condição primordial para a produção de commodities. Falhas e erros na aplicação de agrotóxicos têm passado despercebidos e, assim como outros problemas de condução de lavouras, têm sido compensados pela expansão de áreas e produção. O crescimento territorial quase estagnado nas regiões agrícolas

\footnotetext{
${ }^{1}$ Prof. Doutor, Departamento de Agronomia, Universidade Estadual de Maringá, Av. Colombo, 5790, Maringá - PR, constantin@teracom.com.br, jgcsales@uem.br, cdgmaciel2@uem.br 
faz com que a condução das lavouras e em especial as aplicações tornem-se mais profissionais (CABEDA, 2004). Nesse sentido, a tecnologia de aplicação idealiza a interação de vários fatores que maximizarão a eficácia, a economicidade e a operacionalidade dos tratamentos, através da adequação de máquinas, da menor contaminação ambiental e da segurança do operador.

Segundo FARINHA et al. (2009a, b), os incrementos da área cultivada e produção de soja no Brasil, nos últimos 32 anos, estimularam o uso de tecnologias de aplicação de produtos fitossanitários mais acuradas. O sucesso da aplicação e o resultado do controle estão diretamente relacionados aos fatores: seleção de pontas, ajuste do volume da calda, parâmetros operacionais, condições ambientais favoráveis e momento correto da aplicação. Para isso, a definição do depósito em alvos biológicos e a escolha das pontas de pulverização adequadas são fundamentais, visto que interferirá na vazão, cobertura do alvo e uniformidade de distribuição da calda (FERNANDES et al., 2007; NASCIMENTO et al., 2009; ROMÁN et al., 2009).

Existem vários tipos de pontas hidráulicas disponíveis no mercado, com usos definidos para diferentes condições. Entre as mais utilizadas, destacam-se as de jato cônico vazio e as de jato plano, as quais, dependendo da pressão e do ângulo de abertura, podem potencializar muita deriva. Para contornar esse problema, alguns fabricantes oferecem pontas com potencial antideriva (CUNHA et al., 2005a). De maneira geral, recomenda-se, para produtos de contato ou com menor ação sistêmica, o uso de gotas menores e/ou maior volume de calda, devido à maior dependência desta técnica com relação à cobertura dos alvos. Para produtos sistêmicos, pode ser utilizada menor densidade de gotas, o que facilita a adoção de técnicas para redução de deriva através de gotas maiores, assim como melhora a segurança da aplicação e a eficiência operacional (ANTUNIASSI, 2010).

Ao estudar o efeito da eletrização de gotas sobre a variabilidade dos depósitos de pulverização, SOUZA et al. (2007a) concluiu que a irregularidade da deposição pode levar à necessidade de aumento da dose aplicada e que a proximidade das plantas daninhas com a cultura da soja reduz a uniformidade e os depósitos da calda de aplicação. Recentemente, vários trabalhos têm sido desenvolvidos analisando o desempenho de pontas de pulverização associadas à redução de volumes de calda, buscando melhorias na capacidade de trabalho e rendimento operacional das aplicações no controle de pragas e doenças, na cultura da soja em estádios reprodutivos. Entretanto, o aprimoramento da tecnologia de aplicação visando a reduzir o volume de calda tem como consequência negativa a diminuição da cobertura adequada do alvo desejado, principalmente nas partes inferiores da cultura (SCUDELER et al., 2004; CUNHA et al., 2006; CUNHA et al., 2008; BOSCHINI et al., 2008; FARINHA et al., 2009a; NASCIMENTO et al., 2009; BAUER et al., 2009; CUNHA \& PERES, 2010; CHRISTOVAM et al., 2010a, b).

Com referência a estágios vegetativos, trabalhos que abordam tecnologias de aplicação de agrotóxico em soja, normalmente, visam a quantificar a eficácia da deposição da calda de pulverização sobre a cultura e/ou plantas daninhas, comparando o desempenho de equipamentos, as condições operacionais de trabalho, os modelos de pontas de pulverização e a ação de adjuvantes.

Desta forma, o presente trabalho tem como objetivo avaliar a deposição da calda de pulverização, produzida por diferentes modelos de pontas de pulverização e pressões de trabalho, no estádio fenológico $\mathrm{V}_{6}$ da cultura da soja.

\section{MATERIAL E MÉTODOS}

O experimento foi desenvolvido em campo de produção de soja, cultivar CODETEC 206, na Fazenda Experimental da Universidade Estadual de Maringá, localizada no distrito de Iguatemi, pertencente ao Município de Maringá - PR, no ano agrícola de 2004/2005. O delineamento experimental utilizado foi o inteiramente casualizado, em esquema fatorial 4x2, com oito tratamentos e cinquenta repetições, caracterizadas por trifólios de soja. Os tratamentos foram representados por quatro modelos de pontas de pulverização do fabricante Magno ${ }^{\circledR}$ e duas condições de pressão de trabalho $(207 \mathrm{e} 414 \mathrm{kPa})$, constituindo: AD $11002\left(152 \mathrm{~L} \mathrm{ha}^{-1} \mathrm{e}\right.$ 
$\left.208 \mathrm{~L} \mathrm{ha}^{-1}\right)$; AD/D 11002 (152 $\mathrm{L} \mathrm{ha}^{-1}$ e $\left.208 \mathrm{~L} \mathrm{ha}^{-1}\right)$; AD-IA/D $11002\left(152 \mathrm{~L} \mathrm{ha}^{-1}\right.$ e $\left.208 \mathrm{~L} \mathrm{ha}^{-1}\right)$; MAG 2 e MAG 3 (157 L ha ${ }^{-1}$ e $212 \mathrm{~L} \mathrm{ha}^{-1}$ ).

As variáveis respostas avaliadas foram o monitoramento e a quantificação da deposição da calda de pulverização nas porções superior e inferiores das plantas de soja em estádio fenológico $\mathrm{V}_{6}$. Considerou-se como alvos naturais os mesmos trifólios foliares para deposição da calda de pulverização formadas pelas duas pressões de trabalho para cada um dos modelos de pontas de pulverização estudadas. Como traçadores da deposição da pulverização, foram utilizados na calda de pulverização, separadamente, os corantes alimentícios Azul Brilhante FD\&C-1 (0,3\% p/v) para os quatro modelos de pontas em pressão de $207 \mathrm{kPa}$ e Amarelo de Tartrasina FD\&C-5 (0,6\% p/v) para as mesmas pontas em pressão de $414 \mathrm{kPa}$, conforme adaptações das metodologias descritas por SOUZA et al. (2007a) e MACIEL et al. (2007). Em todos os tratamentos, adicionou-se nas soluções traçadoras o espalhante adesivo Assist ${ }^{\circledR}$ (Basf do Brasil S/A), na dose de 0,5\% (v/v), assim como foi utilizado o intervalo de aproximadamente 30 minutos entre a aplicação de um traçante e o outro sobre os mesmos alvos.

A aplicação dos tratamentos foi realizada utilizando-se de um pulverizador costal, com pressão constante, à base de $\mathrm{CO}_{2}$, equipado com reservatório de três litros e barra de quatro bicos espaçados de $50 \mathrm{~cm}$, com pontas posicionadas a $50 \mathrm{~cm}$ de altura das plantas. Os tratamentos foram efetuados através de um conjunto aplicador+pulverizador+trator, na velocidade de trabalho de $5,5 \mathrm{~km} \mathrm{~h}^{-1}$, conforme ilustrado na Figura 1. No momento das aplicações (21/12/2004), as condições climáticas de temperatura, umidade relativa do ar e velocidade dos ventos, em leitura tomada entre as aplicações, foram de $26,3^{\circ} \mathrm{C}, 69 \%$ e $5,1 \mathrm{~km} \mathrm{~h}^{-1}$, respectivamente.

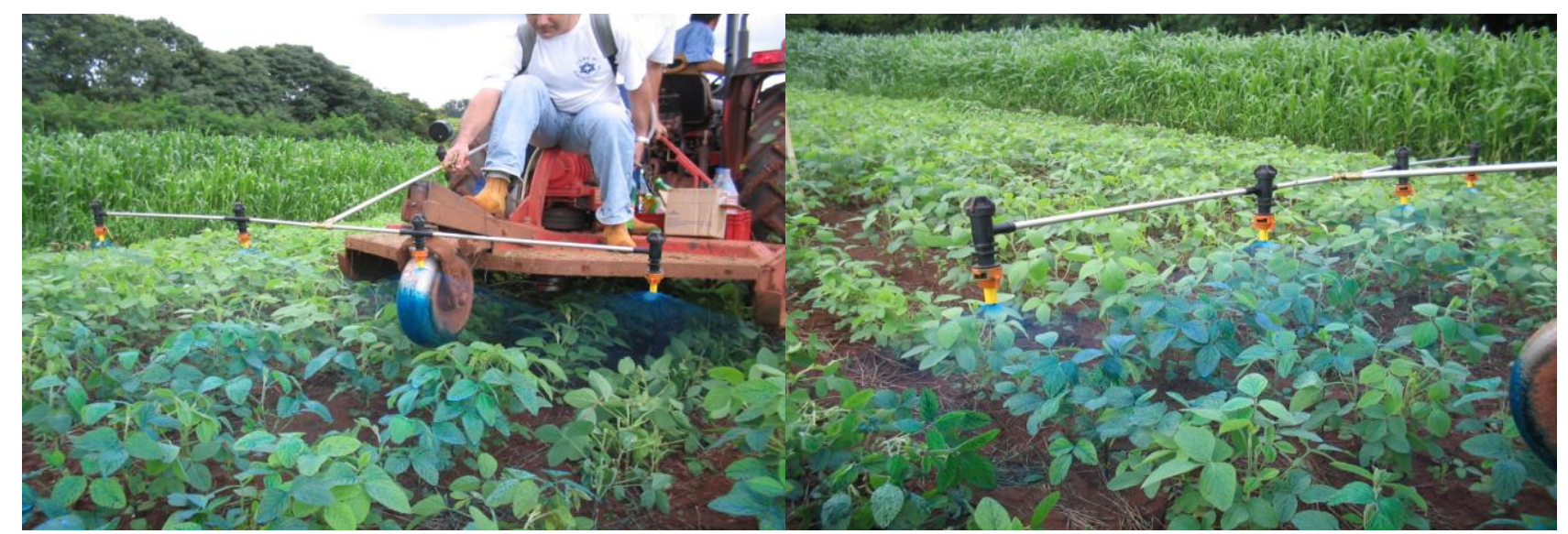

FIGURA 1. Representação esquemática da dinâmica da pulverização dos traçantes na cultura da soja "cultivar CODETEC 206" no estádio fenológico $\mathrm{V}_{6}$. Schematic representation of tracers spray dynamic in soybean crop "CODETEC 206 genotype" in $V_{6}$ grow stage.

O procedimento de recuperação das soluções traçadoras constituídas pelas caldas de pulverização foi desenvolvido através da lavagem dos trifólios-alvos, com volumes de $50 \mathrm{~mL}$ de água destilada em sacos plásticos, através de agitação constante dos mesmos, por 20 segundos. A determinação das quantidades dos traçantes depositadas, em cada amostra, foram realizadas utilizando-se de procedimentos de espectrofotometria. Os resultados em absorbância das leituras, nos comprimentos de onda a $630 \mathrm{~nm}$ e $427 \mathrm{~nm}$, foram realizados respectivamente, com os traçantes FD\&C-1 e FD\&C-5, e transformados em mg L ${ }^{-1}$, de acordo com coeficiente angular da curva-padrão, similarmente à metodologia utilizada por SOUZA et al. (2007a,b).

As concentrações dos depósitos foram transformadas em volume por área $\left(\mu \mathrm{L} \mathrm{cm}^{-2}\right)$, após a determinação da área foliar dos trifólios e pecíolos totalmente expandidos com auxílio de um integrador de área foliar (areameter), modelo LI 3100 ${ }^{\circledR}$. Posteriormente, os resultados da deposição foram ajustados ao modelo de Gompertz, utilizando-se do programa estatístico SAS. Os respectivos 
coeficientes de determinação, frequências acumuladas e não acumuladas (derivada da primeira da do modelo de Gompertz = F'), assim como suas modas e medianas da deposição das caldas pulverizadas foram representados para as condições estudadas, conforme VELINI (1995).

Modelo de Gompertz: $\mathrm{F}=\mathrm{e}^{\wedge}\left(\mathrm{a}-\mathrm{e}^{\wedge}(-\mathrm{b}-\mathrm{c} \mathrm{x})\right)$

em que,

F - frequência acumulada, em porcentagem;

a - valor atribuído em 4,605170, de modo que e $\mathrm{a}=100$;

b - valor estimado pelo modelo;

c - valor estimado pelo modelo, e

$\mathrm{x}$ - depósitos, em $\mu \mathrm{L} \mathrm{cm}{ }^{-2}$.

Os valores modais foram determinados através da derivada da primeira do modelo de Gompertz $\left(F^{\prime}=c \quad e^{\wedge}\left[a-b-c \quad x-e^{\wedge}(-b-c x)\right]\right)$ e suas medianas calculadas, igualando-se à própria expressão, que representam as frequências acumuladas a 50\% (densidade de probabilidade).

Os depósitos obtidos através do contraste entre as variáveis pontas de pulverização e pressão de trabalho também foram analisados em esquema fatorial $5 \times 2$, através de análise de variância pelo teste $\mathrm{F}$, e suas médias, comparadas pelo teste de Tukey, a $5 \%$ de probabilidade.

\section{RESULTADOS E DISCUSSÃO}

Os resultados apresentados nas Figuras 2; 3; 4 e 5, correspondentes aos alvos trifólios foliares coletados nas posições superior e inferior das plantas de soja em estádio $\mathrm{V}_{6}$, representam, no eixo Y1 (escala à esquerda), a frequência acumulada, ou seja, os valores medianos para as diferentes porcentagens de depósitos da calda de pulverização amostrados e a frequência não acumulada, no eixo Y2 (escala à direita), pela derivada da primeira do modelo. Para facilitar a visualização e a interpretação das informações que foram adequadamente ajustadas ao modelo de Gompertz, VELINI (1995) relata que a concavidade da curva demonstra a uniformidade da deposição da calda pulverizada, onde quanto mais plana a curva, maior é a frequência de valores extremos, o que caracteriza maior amplitude de depósito na amostragem.

Em estudos comparativos, utilizando-se de traçantes na calda de pulverização, é fundamental a padronização da área foliar ao utilizar o mesmo alvo para compreensão de variáveis diferentes, como foi realizado neste estudo, onde, para as variáveis pressões 207 e $414 \mathrm{kPa}$, foram pulverizados traçadores distintos sobre as mesmas plantas de soja.

Nas Figuras 2; 3; 4 e 5, observa-se que, independentemente das pontas e da pressão estudada, houve maior irregularidade na quantidade depositada pela pulverização nos trifólios superiores em relação à porção inferior das plantas de soja em estádio $\mathrm{V}_{6}$, representado pelas disposições mais planas das curvas ajustadas ao modelo de Gompertz ou, ainda, através do parâmetro "C" do modelo (Tabela 1). Apesar de a maior uniformidade da deposição da calda pulverizada ter sido determinada na porção inferior das plantas (menores inclinações nas curvas e/ou maiores valores de parâmetro “C”), independentemente da ponta ou pressão estudada, as quantidades médias, modais e medianas expressas por unidade de área foram inferiores às encontradas na porção superior das plantas. Entretanto, o melhor desempenho na dinâmica de deposição, assim como na capacidade de penetração das gotas pulverizadas na região basal da soja, foi obtido com a ponta MAG 2 (414 kPa e $157 \mathrm{~L} \mathrm{ha}^{-1}$ ), a qual apresentou valores de média, moda e mediana superiores aos modelos AD/D 110.02, AD-IA 110.02 e AD $110.02\left(207 \mathrm{kPa}\right.$ e $\left.152 \mathrm{~L} \mathrm{ha}^{-1}\right)$. Esses resultados corroboram os de SCUDELER et al. (2004) e ANTUNIASSI (2010), os quais avaliando a cobertura de folhas de soja com diferentes pontas de pulverização, concluíram que gotas finas propiciam melhores coberturas nas posições média e baixa das plantas. Para BOSCHINI et al. (2008), a ponta de pulverização que apresentou maiores deposições, no extrato superior das plantas de soja, foi o modelo jato plano duplo com volumes de calda de $300 \mathrm{~L} \mathrm{ha}^{-1}$ e, para o extrato mediano e inferior, o jato cone vazio com volumes de calda de 200 ou $300 \mathrm{~L} \mathrm{ha}^{-1}$. 

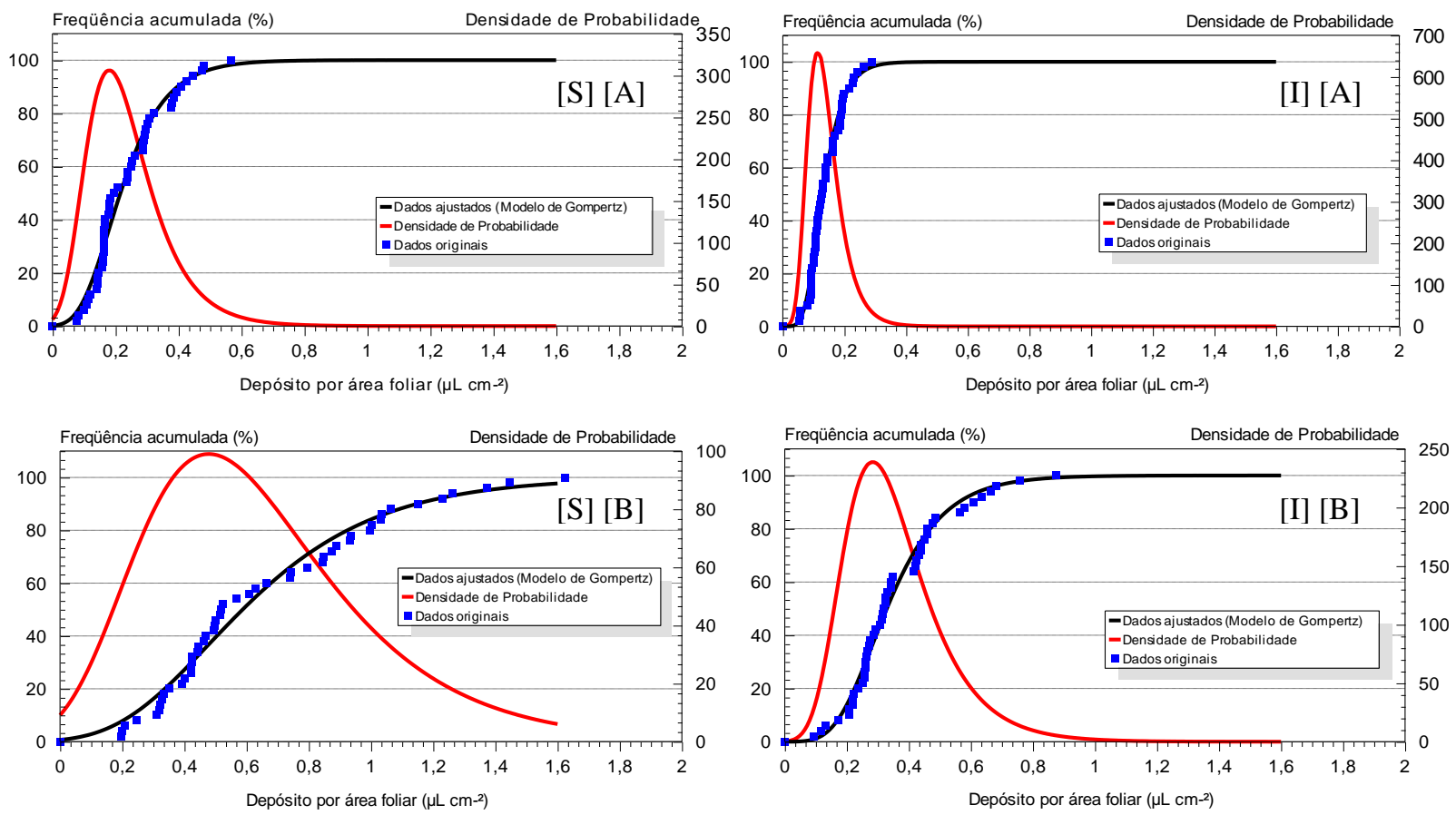

FIGURA 2. Frequências acumuladas (\%) e densidades de probabilidade relativas à deposição da calda de pulverização por unidade de área foliar das porções superiores [S] e inferiores [I] da cultura da soja, em estádio $\mathrm{V}_{6}$, utilizando-se da ponta $\mathrm{AD} 110.02 \mathrm{em}$ pressão de $207 \mathrm{kPa}$ $\left(152 \mathrm{~L} \mathrm{ha}^{-1}\right)$ [A] e $414 \mathrm{kPa}\left(208 \mathrm{~L} \mathrm{ha}^{-1}\right)$ [B], através do modelo de Gompertz. Accumulated frequencies $(\%)$ and probability densities relative to spray deposition per leaves area unit of superior [s] and inferior [I] portions of soybean crop $\mathrm{V}_{6}$ grow stage, by using AD 110.02 nozzle with $207 \mathrm{kPa}\left(152 \mathrm{~L} \mathrm{ha}^{-1}\right)$ [A] and $414 \mathrm{kPa}\left(208 \mathrm{~L} \mathrm{ha}^{-1}\right)$ [B], pressures through Gompertz model.
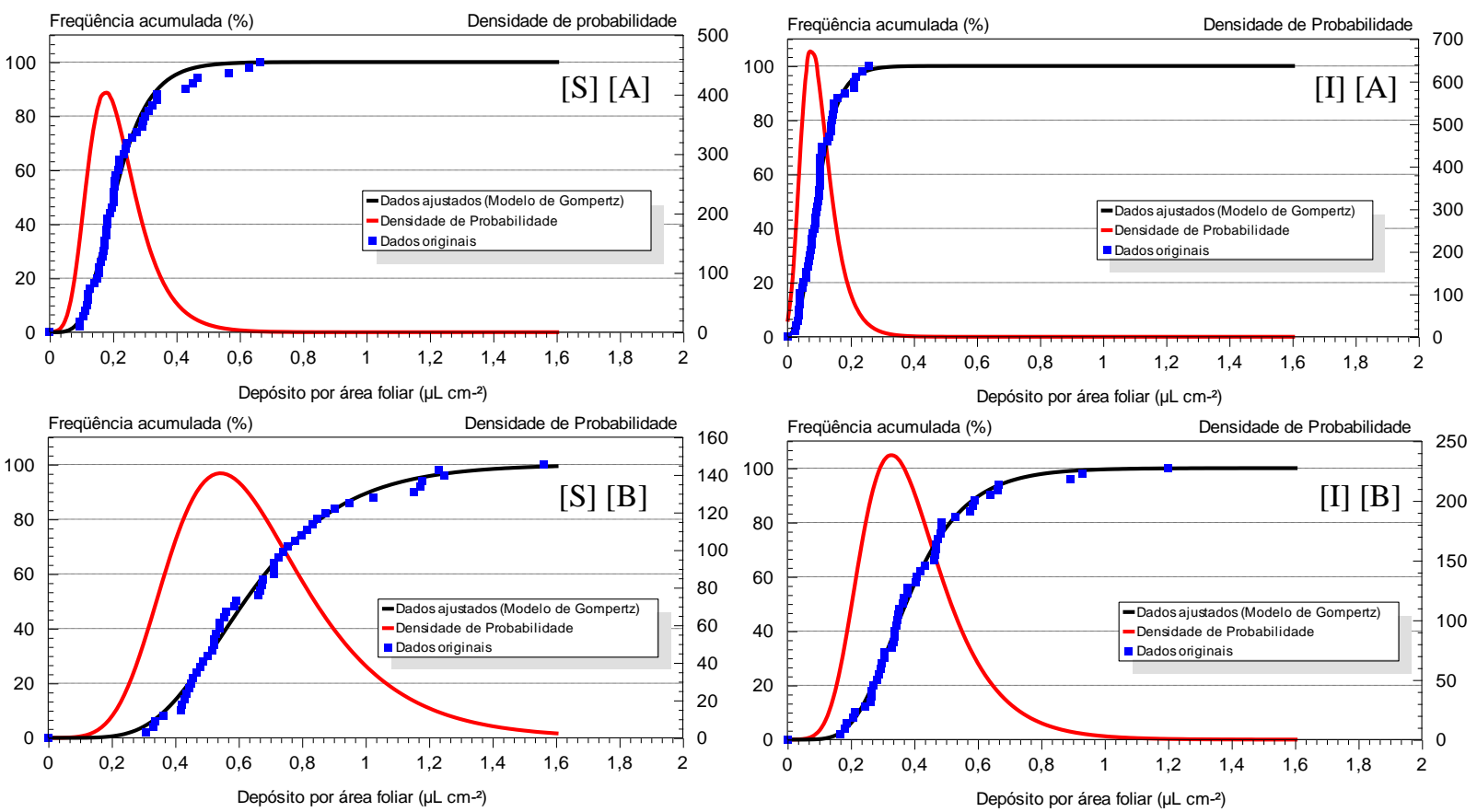

FIGURA 3. Frequências acumuladas (\%) e densidades de probabilidade relativas à deposição da calda de pulverização por unidade de área foliar das porções superiores [S] e inferiores [I] da cultura da soja em estádio $\mathrm{V}_{6}$, utilizando-se da ponta AD/D $110.02 \mathrm{em}$ pressão de $207 \mathrm{kPa}$ $\left(152 \mathrm{~L} \mathrm{ha}^{-1}\right)$ [A] e $414 \mathrm{kPa}\left(208 \mathrm{~L} \mathrm{ha}^{-1}\right)$ [B], através do modelo de Gompertz. Accumulated frequencies $(\%)$ and probability densities relative to spray deposition per leaves area unit of superior [s] and inferior [I] portions of soybean crop $V_{6}$ grow stage, by using AD/D 110.02 nozzle with $207 \mathrm{kPa}\left(152 \mathrm{~L} \mathrm{ha}^{-1}\right)$ [A] and $414 \mathrm{kPa}\left(208 \mathrm{~L} \mathrm{ha}^{-1}\right)$ [B], pressures through Gompertz model. 

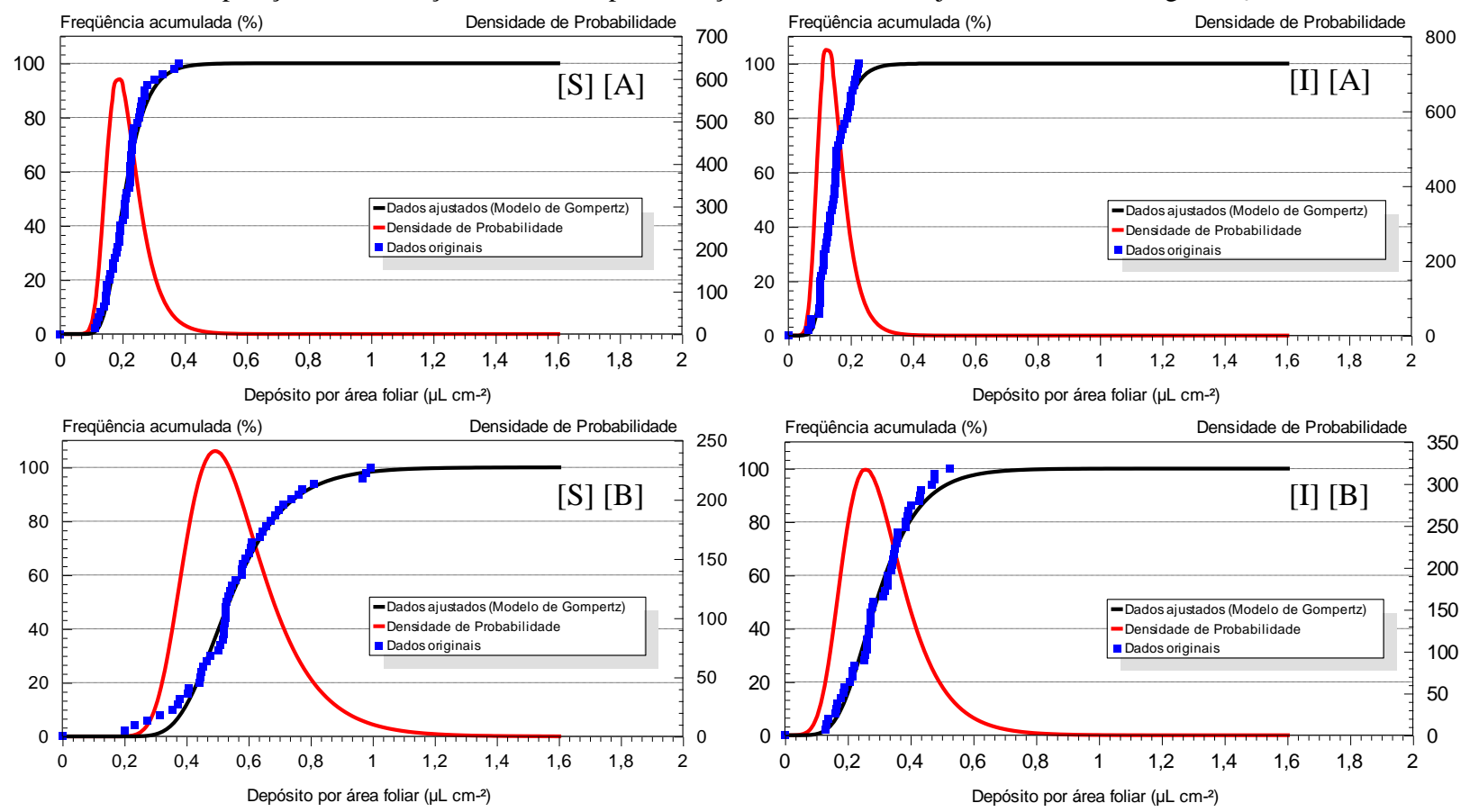

FIGURA 4. Frequências acumuladas (\%) e densidades de probabilidade relativas à deposição da calda de pulverização por unidade de área foliar das porções superiores [S] e inferiores [I] da cultura da soja em estádio $\mathrm{V}_{6}$, utilizando-se da ponta AD-IA/D 11002 em pressão de 207 $\mathrm{kPa}\left(152 \mathrm{~L} \mathrm{ha}^{-1}\right)$ [A] e $414 \mathrm{kPa}\left(208 \mathrm{~L} \mathrm{ha}^{-1}\right)$ [B], através do modelo de Gompertz. Accumulated frequencies $(\%)$ and probability densities relative to spray deposition per leaves area unit of superior [s] and inferior [I] portions of soybean crop $\mathrm{V}_{6}$ grow stage, by using AD-IA/D 110.02 nozzle with $207 \mathrm{kPa}\left(152 \mathrm{~L} \mathrm{ha}^{-1}\right)$ [A] and $414 \mathrm{kPa}(208$ $\mathrm{L} \mathrm{ha}^{-1}$ ) [B], pressures through Gompertz model.
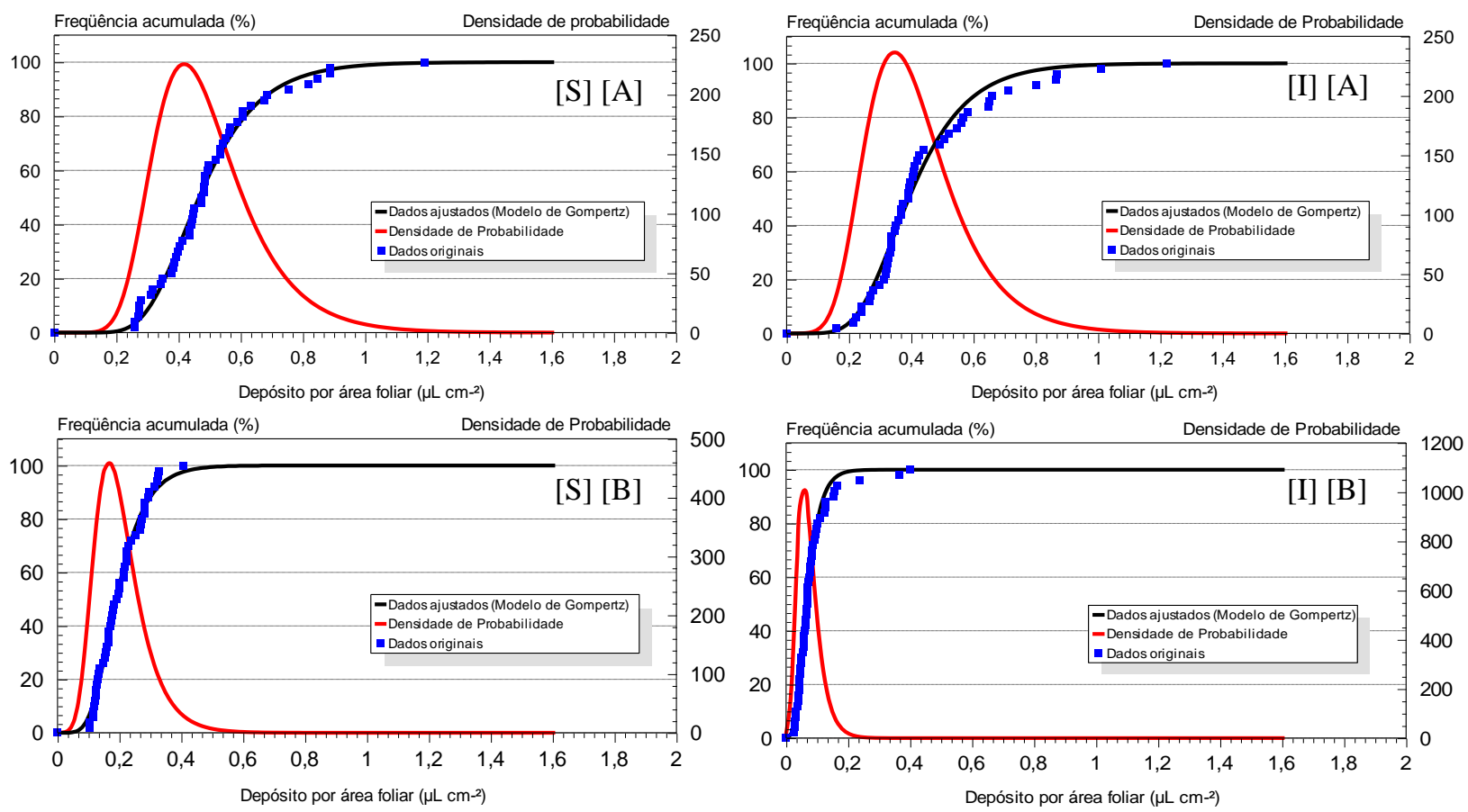

FIGURA 5. Frequências acumuladas (\%) e densidades de probabilidade relativas à deposição da calda de pulverização por unidade de área foliar das porções superiores $[S]$ e inferiores $[\mathrm{I}]$ da cultura da soja em estádio $V_{6}$, utilizando-se das pontas MAG 2 e MAG 3 em pressão de 414 $\mathrm{kPa}\left(157 \mathrm{~L} \mathrm{ha}^{-1}\right)[\mathrm{A}]$ e $\left(212 \mathrm{~L} \mathrm{ha}^{-1}\right)$ [B], através do modelo de Gompertz. Accumulated frequencies $(\%)$ and probability densities relative to spray deposition per leaves area unit of superior [s] and inferior [I] portions of soybean crop $V_{6}$ grow stage, by using MAG 2 and MAG 3nozzles with $414 \mathrm{kPa}\left(157 \mathrm{~L} \mathrm{ha}^{-1}\right)$ [A] and $\left(212 \mathrm{~L} \mathrm{ha}^{-1}\right)$ [B], pressure through Gompertz model. 
TABELA 1. Resultados das análises de regressão entre as frequências acumuladas e os volumes de depósito das soluções traçadoras pulverizadas em pressão de trabalho com 207 e 414 $\mathrm{kPa}$, utilizando o modelo de Gompertz, modas e medianas da deposição nas posições superior e inferior das plantas de soja, em estádio $\mathrm{V}_{6}$. Results of regression analysis between accumulated frequencies and volumes of sprayed tracers solutions with 207 e $414 \mathrm{kPa}$ work pressures, by using Gompertz model, modal and median of spray deposition in superior and inferior portions of soybean plants $V_{6}$ grow stage.

\begin{tabular}{|c|c|c|c|c|c|}
\hline \multirow{4}{*}{ Modelo } & \multicolumn{5}{|c|}{ Frequência Acumulada $(\%)=\mathrm{e}^{\wedge}\left(\mathrm{a}-\mathrm{e}^{\wedge}(-\mathrm{b}-\mathrm{c} \mathrm{X})\right)$} \\
\hline & & \multicolumn{4}{|c|}{ Depósito $\left(\mu \mathrm{L} \mathrm{cm}^{-2}\right)$ - Porção Superior } \\
\hline & & AD 11002 & AD/D 11002 & AD-IA/D 11002 & MAG 2 \\
\hline & & $(207 \mathrm{kPa})$ & $(207 \mathrm{kPa})$ & $(207 \mathrm{kPa})$ & $(414 \mathrm{kPa})$ \\
\hline Estimativa & A & 4,60517 & 4,60517 & 4,60517 & 4,60517 \\
\hline Dos & $\mathrm{B}$ & $-1,87928$ & $-2,42548$ & $-3,83000$ & $-3,20568$ \\
\hline Parâmetros & $\mathrm{C}$ & 8,31741 & 10,96557 & 16,36704 & 6,13200 \\
\hline F Regressão & & $85360,76 * *$ & $85588,25 * *$ & $85434,15 * *$ & $85672,79 * *$ \\
\hline S Q Resíduo & & 978,48 & 523,51 & 831,70 & 354,41 \\
\hline $\mathrm{R}^{2}$ & & 0,9779 & 0,9881 & 0,9812 & 0,9820 \\
\hline Moda & & 0,2259 & 0,2212 & 0,2340 & 0,5228 \\
\hline Mediana & & 0,2700 & 0,2546 & 0,2564 & 0,5825 \\
\hline & & \multicolumn{4}{|c|}{ Depósito $\left(\mu \mathrm{L} \mathrm{cm}^{-2}\right)$ - Porção Inferior } \\
\hline Estimativa & A & 4,60517 & 4,60517 & 4,60517 & 4,60517 \\
\hline Dos & $\mathrm{B}$ & $-2,53917$ & $-1,74131$ & $-3,29101$ & $-2,78659$ \\
\hline Parâmetros & $\mathrm{C}$ & 17,88148 & 18,31104 & 20,98211 & 6,42778 \\
\hline F Regressão & & $85621,57 * *$ & $85609,73 * *$ & $85627,98 * *$ & $85439,78 * *$ \\
\hline S Q Resíduo & & 456,85 & 480,54 & 444,04 & 820,44 \\
\hline $\mathrm{R}^{2}$ & & 0,9897 & 0,9891 & 0,9899 & 0,9814 \\
\hline Moda & & 0,1420 & 0,0951 & 0,1568 & 0,4335 \\
\hline \multirow[t]{4}{*}{ Mediana } & & 0,1625 & 0,1151 & 0,1743 & 0,4905 \\
\hline & & \multicolumn{4}{|c|}{ Depósito $\left(\mu \mathrm{L} \mathrm{cm}^{-2}\right)$ - Porção Superior } \\
\hline & & AD 11002 & AD/D 11002 & AD-IA/D 11002 & MAG 3 \\
\hline & & $(414 \mathrm{kPa})$ & $(414 \mathrm{kPa})$ & $(414 \mathrm{kPa})$ & $(414 \mathrm{kPa})$ \\
\hline Estimativa & $\mathrm{A}$ & 4,60517 & 4,60517 & 4,60517 & 4,60517 \\
\hline Dos & $\mathrm{B}$ & $-1,60854$ & $-2,59861$ & $-4,03935$ & $-2,59889$ \\
\hline Parâmetros & $\mathrm{C}$ & 2,69037 & 3,82776 & 6,55213 & 12,46859 \\
\hline F Regressão & & $85431,19 * *$ & $85659,59 * *$ & $85549,01 * *$ & $85697,37 * *$ \\
\hline S Q Resíduo & & 837,60 & 380,82 & 601,98 & 305,27 \\
\hline $\mathrm{R}^{2}$ & & 0,9810 & 0,9914 & 0,9899 & 0,9931 \\
\hline Moda & & 0,5979 & 0,6789 & 0,6165 & 0,2084 \\
\hline Mediana & & 0,7341 & 0,7746 & 0,6724 & 0,2378 \\
\hline & & \multicolumn{4}{|c|}{ Depósito $\left(\mu \mathrm{L} \mathrm{cm}^{-2}\right)$ - Porção Inferior } \\
\hline Estimativa & A & 4,60517 & 4,60517 & 4,60517 & 4,60517 \\
\hline Dos & B & $-2,30333$ & $-2,65552$ & $-2,77274$ & $-1,95392$ \\
\hline Parâmetros & $\mathrm{C}$ & 6,49041 & 6,47532 & 8,62037 & 27,60870 \\
\hline F Regressão & & $85560,54 * *$ & $85718,82 * *$ & $85449,49 * *$ & $85673,59 * *$ \\
\hline S Q Resíduo & & 578,92 & 262,35 & 801,03 & 352,82 \\
\hline $\mathrm{R}^{2}$ & & 0,9869 & 0,9941 & 0,9819 & 0,9920 \\
\hline Moda & & 0,3548 & 0,4101 & 0,3216 & 0,0707 \\
\hline Mediana & & 0,4113 & 0,4667 & 0,3642 & 0,0840 \\
\hline
\end{tabular}


A diferença dos valores modais (maior frequência de valores) para a deposição da calda de pulverização da ponta MAG $2\left(414 \mathrm{kPa}\right.$ e $\left.157 \mathrm{~L} \mathrm{ha}^{-1}\right)$, na porção inferior em relação à superior da cultura soja, foi de 17,1\% (Tabela 1). Esse valor indica ter sido pequena a razão entre as frequências da deposição nas regiões superior e inferior da planta, caracterizando uma deposição mais uniforme em relação à planta total, quando comparada com as pontas AD-IA/D 11002, AD 11002 e AD/D 11002 (207 kPa e $\left.152 \mathrm{~L} \mathrm{ha}^{-1}\right)$, os quais, em média, apresentaram diferenças significativas em torno de 33,0\%, 37,1\% e 57,0\%, respectivamente. Além disso, a ponta MAG 2 também apresentou deposição da pulverização nos trifólios inferiores das plantas 83,9\% maior do que a MAG 3 (414 $\mathrm{kPa}$ e $212 \mathrm{~L} \mathrm{ha}^{-1}$ ). Este fato pode ser explicado pela possibilidade de ter havido perdas acentuadas por deriva e evaporação na pulverização com a ponta MAG 3, uma vez que o aumento da pressão de trabalho modifica o espectro de gotas produzidas na aspersão, favorecendo a redução do tamanho das gotas para pontas tipo cone vazio, conforme informações de RAMOS \& PIO (2008).

Esses resultados não corroboram os descritos por CUNHA et al. (2005a,b), CUNHA et al. (2008) e BAUER et al. (2008), em que não observaram influência das pontas jato plano padrão, jato plano antideriva e cônico vazio na aplicação de fungicida para o controle de doenças na cultura do feijoeiro e da soja. Para a cultura da soja em estádio $\mathrm{R}_{2}$, CUNHA et al. (2006) constataram que, apesar de o volume de calda de $160 \mathrm{~L} \mathrm{ha}^{-1}$ e a utilização da ponta de jato plano padrão (API 110.02) terem proporcionado maior uniformidade de distribuição da calda fungicida, também não foi caracterizada influência do tipo de ponta de pulverização e do volume de aplicação no controle da ferrugem asiática e na produtividade da cultura. MADALOSSO (2007) e ROMÁN et al. (2009), trabalhando com pontas de jato cônico vazio e jato plano duplo no conector "DUO", foram similares quanto à porcentagem de cobertura e no volume de aplicação de fungicida na cultura da soja. Entretanto, VIANA et al. (2008), utilizando pontas de jato plano duplo e jato cônico vazio na cultura da soja, verificou melhor resultado de cobertura da pulverização para a ponta de jato plano duplo.

A variação dos níveis de deposição indicados pela inclinação das curvas (Figuras 2; 3; 4 e 5) ou pelo parâmetro "C" do modelo de Gompertz na pressão em $414 \mathrm{kPa}$ (Tabela 1), constatada nos trifólios superiores e inferiores, sugerem a melhor eficiência na uniformidade de distribuição da pulverização para as pontas MAG 2 e MAG 3, em relação a AD-IA/D 11002, AD/D 11002. Em relação à quantidade de deposição da pulverização nos trifólios superiores e inferiores, os valores de moda e mediana apresentaram ordem de eficiência das pontas inversamente proporcional à característica de uniformidade na pressão de $414 \mathrm{kPa}$. Desta forma, os resultados sugerem que todas as pontas-leque foram superiores aos modelos cônicos MAG 2 e MAG 3, e que, apesar de terem apresentado dinâmicas bastante semelhantes, as pontas AD/D 11002 e AD 11002 destacaram-se principalmente na maior deposição nos trifólios superiores e inferiores da soja, em relação ao modelo AD-IA/D 11002.

GAZZIERO et al. (2006) e SOUZA et al. (2007a), ao compararem estádios de desenvolvimento da cultura da soja, observaram decréscimo no depósito e uniformidade da pulverização por unidade de área com o aumento da área foliar, evidenciando que o efeito de redução da deposição está associado ao acúmulo de folhas, devido á sobreposição dos trifólios. Segundo os autores, isso indica que o desenvolvimento da cultura, possivelmente, represente um fator de aumento de seletividade de herbicidas pós-emergentes, devido ao menor volume depositado, principalmente de ativos, cuja dose aplicada representaria o fator de seletividade.

Apesar de consideramos a existência de grande variabilidade na deposição da calda de pulverização, conforme VELINI (2004), essa dispersão, associada à escolha indevida do método de análise, pode dificultar a interpretação dos resultados e praticamente impossibilitar conclusões confiáveis. Ainda assim, os valores médios dos depósitos foram representados na Tabela 3, contrastando com os modelos de pontas e pressões estudados em esquema fatorial, e as informações obtidas corroboraram e consolidaram os resultados anteriormente descritos. 
Em termos de valores médios de depósito (Tabela 2), a ponta MAG 2 (414 kPa e $157 \mathrm{~L} \mathrm{ha}^{-1}$ ) superou os modelos-leque na pressão de $207 \mathrm{kPa}$ e da MAG 3 (414 kPa e $212 \mathrm{~L} \mathrm{ha}^{-1}$ ), independentemente do posicionamento dos trifólios da soja. As pontas AD/D 11002, AD 11002 e AD-IA/D 11002 (208 L ha $\left.{ }^{-1}\right)$, em pressão de $414 \mathrm{kPa}$, foram significativamente superiores a MAG 2 e MAG 3 independentemente da posição dos trifólios da soja, e quando comparado a seus próprios modelos em $207 \mathrm{kPa}$, promoveram aumento médio da deposição na ordem de 63,5\% e $64,1 \%$, respectivamente, sobre os trifólios superiores e inferiores. A análise em termos médios, de forma semelhante às frequências quantitativas das distribuições, caracteriza a superioridade da deposição da calda aplicada para a ponta MAG 2 em baixa pressão (207 kPa) e dos modelos-leque $\mathrm{AD} / \mathrm{D} 11002$ e AD 11002 em alta pressão (414 kPa), independentemente da posição dos trifólios foliar da soja em estádio fenológico $\mathrm{V}_{6}$. BOLLER \& MACRHY (2007), avaliando a eficácia do herbicida bentazon, aplicado em diferentes pressões de trabalho, constataram que a ponta anti deriva da série AI 110015 proporcionou níveis inferiores de controle de picão-preto (Bidens spp.), em relação aos modelos XR 110015, DG 110115 e TT 110015, que não diferiram estatisticamente entre si.

TABELA 2. Deposição média da calda de pulverização nas porções superior e inferior das plantas de soja em estádio $\mathrm{V}_{6}$, contrastando com os modelos de ponta e pressões estudadas. Medium spray depositions over superior and inferior portions of soybean plants $V_{6}$ grow stage, contrasting the spray nozzle models and studied pressures.

\begin{tabular}{|c|c|c|c|c|}
\hline \multirow{3}{*}{ Tratamentos } & \multicolumn{4}{|c|}{ Depósito $\left(\mu \mathrm{L} \mathrm{cm}^{-2}\right)$ - Posicionamento } \\
\hline & \multirow{2}{*}{$\begin{array}{l}\text { Superior } \\
207 \mathrm{kPa}\end{array}$} & \multirow{2}{*}{$\begin{array}{l}\text { Superior } \\
414 \mathrm{kPa}\end{array}$} & \multirow{2}{*}{$\begin{array}{l}\text { Inferior } \\
207 \mathrm{kPa}\end{array}$} & \multirow{2}{*}{$\begin{array}{l}\text { Inferior } \\
414 \mathrm{kPa}\end{array}$} \\
\hline & & & & \\
\hline 1.) $\mathrm{AD} 11002$ & 0,3037 b B & 0,8364 a $\mathrm{A}$ & 0,1764 b B & 0,4577 b A \\
\hline 2.) $\mathrm{AD} / \mathrm{D} 11002$ & 0,2998 b B & 0,8553 a $A$ & 0,1289 c $\mathrm{B}$ & 0,5246 a $A$ \\
\hline 3.) $\mathrm{AD}-\mathrm{IA} / \mathrm{D} 11002$ & 0,2659 b B & $0,6983 \mathrm{~b} \quad \mathrm{~A}$ & 0,1820 b B & 0,3777 c $\mathrm{A}$ \\
\hline 4.) MAG 2 e MAG 3 & 0,6301 a $\mathrm{A}$ & 0,2550 c $B$ & 0,5645 a $\mathrm{A}$ & $0,1092 \mathrm{~d} \mathrm{~B}$ \\
\hline$\overline{\text { Ponta }(\mathrm{P})}$ & \multicolumn{2}{|c|}{$25,59 * *$} & \multicolumn{2}{|c|}{7,33 ** } \\
\hline Pressão (OS) & \multicolumn{2}{|c|}{$475,42 * *$} & \multicolumn{2}{|c|}{$129,20 * *$} \\
\hline $\mathrm{P} \times \mathrm{OS}$ & \multicolumn{2}{|c|}{$285,92 * *$} & \multicolumn{2}{|c|}{$432,79 * *$} \\
\hline$\overline{\text { C.V.\% }}$ & \multicolumn{2}{|c|}{25,35} & \multicolumn{2}{|c|}{29,13} \\
\hline DMS de $\mathrm{P}(5 \%)$ & \multicolumn{2}{|c|}{0,0678} & \multicolumn{2}{|c|}{0,0474} \\
\hline DMS de OS $(5 \%)$ & \multicolumn{2}{|c|}{0,0258} & \multicolumn{2}{|c|}{0,0361} \\
\hline
\end{tabular}

Obs.: - As pontas MAG 2 e MAG 3 foram utilizadas em $414 \mathrm{kPa}$ - Médias seguidas de mesma letra maiúscula na coluna e minúscula na linha não diferem estatisticamente entre si, pelo teste de Tukey, a $5 \%$ de probabilidade. - ** $=\mathrm{P}<0,01 \mathrm{e} *=\mathrm{P}<0,05$.

Com base nos dados originais, a discrepância entre os maiores e menores depósitos por unidade de área foliar, em função das diferentes pontas e pressões de trabalho, foram pouco representativos para $207 \mathrm{kPa}$ e de maior intensidade em $414 \mathrm{kPa}$, principalmente para as pontas AD 11002, MAG 2 e MAG 3. Para as pressões de 207 e $414 \mathrm{kPa}$, as relações entre maiores e menores depósitos nos trifólios superiores das plantas foram na ordem de 7,4 e 8,3; 7,0 e 5,1; 3,4 e 5,0; 4,6 e 3,9 vezes para as pontas AD 11002; AD/D 11002; AD-IA/D 11002 e MAG (2 e 3), respectivamente, assim como para os trifólios inferiores de 5,5 e 9,5; 10,3 e 7,2; 3,4 e 4,1; 7,7 e 15,0 para as pontas AD 11002; AD/D 11002; AD-IA/D 11002 e MAG (2 e 3). Verifica-se que o aumento da pressão de pulverização de 207 para $414 \mathrm{kPa}$ proporcionou redução da relação entre os maiores e menores depósitos de calda de pulverização para as pontas AD/D 11002 (posição superior e inferior) e MAG 2 (posição superior), condição essa que favorece o aspecto de uniformidade da calda pulverizada em atingir o alvo de forma mais homogênea. Estes resultados corroboram os observados por RODRIGUES-COSTA et al. (2010) e RODRIGUES et al. (2010), em que, independentemente do modelo da ponta de pulverização utilizada, os maiores volumes de aplicação (200 $\mathrm{L} \mathrm{ha}^{-1}$ ) também proporcionaram os maiores depósitos médios e pontuais sobre as espécies feijão (Phaseolus vulgaris), capim-marmelada (Brachiaria plantaginea) e picão-preto (Bidens pilosa) e trapoeraba (Commelina benghalensis). 
Com base nos resultados obtidos, poder-se-ia exemplificar, que se a dose de inseticida ou de fungicida mínima necessária para matar ou proteger contra algum inseto ou doença estivesse contida em $0,2 \mu \mathrm{L} \mathrm{cm}^{-2}$ da área foliar dos trifólios, na região inferior das plantas (Figuras 2; 3; $4 \mathrm{e}$ 5), haveria $70,2 \%$ e $6,5 \% ; 86,4 \%$ e $2,0 \% ; 67,0 \%$ e $5,8 \% ; 1,1 \%$ e $97,2 \%$ de escape de controle para as pontas AD 11002; AD/D 11002; AD-IA/D 11002; MAG (2 e 3), respectivamente, utilizando-se de pressões de 207 e $414 \mathrm{kPa}$. Neste exemplo, observa-se que o aumento da pressão de 207 para $414 \mathrm{kPa}$ promoveria considerável aumento na deposição da pulverização dos defensivos agrícolas sobre os trifólios inferiores nas plantas, na ordem de $63,7 \%, 84,4 \%$ e $61,2 \%$ utilizando-se das pontas AD 11002; AD/D 11002 e AD-IA/D 11002, respectivamente, e redução na deposição na ordem de 96,1\% para MAG 3 em relação a MAG 2, ambas em pressão de trabalho de $414 \mathrm{kPa}$. Em geral, gotas finas produzidas por pontas jato cônico vazio estão mais propícias à perda por deriva $\mathrm{e}$ evaporação, reduzindo a eficiência de deposição da aplicação e aumentando os riscos de contaminação ambiental (SOUZA et al., 2007b; BALAN et al., 2008; QUEIROZ, 2009), principalmente sob condições adversas de temperatura e umidade relativa do ar.

Os resultados ressaltam comportamentos distintos das pontas de pulverização em relação à formação das gotas e volume da aplicação, os quais influenciam na capacidade de penetração da pulverização nas regiões inferiores das plantas de soja, em estádio fenológico $\mathrm{V}_{6}$, e, consequentemente, na eficácia dos defensivos agrícolas em atuar sobre possíveis pragas, doenças e plantas daninhas. Nesse sentido, as pontas MAG 2 e AD/D 11002, na pressão de $414 \mathrm{kPa}(157 \mathrm{e}$ $208 \mathrm{~L} \mathrm{ha}^{-1}$ ), destacam-se pelos melhores desempenhos nas dinâmicas de deposição das pulverizações com resultados superiores as pontas AD 11002 e AD-IA/D 11002 (207 kPa e $152 \mathrm{~L} \mathrm{ha}^{-1}$ ) e MAG 3 (414 kPa e $212 \mathrm{~L} \mathrm{ha}^{-1}$ ), para trifólios das porções superiores e inferiores das plantas. Em contrapartida, as pontas MAG 2 e AD/D 11002 apresentaram como aspecto negativo menor uniformidade da distribuição dos depósitos da calda de pulverização. FARINHA et al. (2009b) obtiveram dados concordantes a esses resultados em que se ressalta a contradição da ocorrência dos tratamentos com maior deposição da calda de pulverização nas plantas de soja terem sido os de menor uniformidade de distribuição. Entretanto, BARCELLOS et al. (2005) relataram que a simples variação da ponta de pulverização e/ou do volume aplicado não se constituiu em medida suficiente para alterar o controle das plantas daninhas, a fitotoxicidade, a altura de plantas e o rendimento de grãos em soja.

\section{CONCLUSÕES}

As maiores quantidades de deposição da calda de pulverização, considerando as posições superior e inferior da cultura da soja no estádio fenológico $\mathrm{V}_{6}$, foram obtidas com as pontas MAG 2 e AD/D 11002, ambos utilizando-se da pressão de trabalho de $414 \mathrm{kPa}$.

O aumento da pressão de 207 para $414 \mathrm{kPa}$, utilizando-se das pontas AD 11002; AD/D 11002 e AD-IA/D 11002, aumentou a deposição da calda de pulverização nos trifólios superiores e inferiores da soja, no estádio fenológico $\mathrm{V}_{6}$, ao contrário do uso da ponta MAG 3 em relação a MAG 2, ambas em pressão de trabalho de $414 \mathrm{kPa}$.

\section{REFERÊNCIAS}

ANTUNIASSI, U.R. Tecnologia de aplicação de defensivos. Rondonópolis: Fundação Mato Grosso, p. 347-372, 2010. (Boletim Técnico de Pesquisa Soja, 14).

BALAN, M.G.; ABI-SAAD; SILVA, C.G.; RIO, A. Deposição da calda pulverizada por três pontas de pulverização sob diferentes condições meteorológicas. Semina: Ciências Agrárias, Londrina, v.29, n.2, p.293-298, 2008.

BARCELLOS, L.C.; ALMEIDA, R.A.; LEÃO, P.G.F.; CARVALHO, Y. Bicos de pulverização na aplicação de herbicidas pós-emergentes na cultura da soja. Pesquisa Agropecuária Tropical, Goiânia, v.35, n.2, p.85-91, 2005. 
BAUER, F.C.; ALMEIDA, E.; MARQUES, D.C.; ROSSI, T.; PEREIRA, F.A.R. Deposição de pontas de pulverização AXI 11002 e JA-2 em diferentes condições operacionais. Ciência Rural, Santa Maria, v.38, n.6, p.1.610-1.614, 2008.

BAUER, F.C.; FERNANDES, C.D.; PEREIRA, F.A.R.; BRIANEZZI FILHO, D.; MARQUES, D.C. Deposição e penetração da calda em cultura de soja (Glycine max (L.) Merrill) com utilização de diferentes volumes de aplicação e assistência de ar. Publicatio UEPG: Ciências Exatas e da Terra, Ciências Agrárias e Engenharias, Ponta Grossa, v.15, n.2, p.107-111, 2009.

BOLLER, W.; MACHRY, M. Efeito da pressão de trabalho e de modelos de pontas de pulverização sobre a eficiência de herbicida de contato em soja. Engenharia Agrícola, Jaboticabal, v.27, n.3, p.722-727, 2007.

BOSCHINI, L.; CONTIERO, R.L.; MACEDO JÚNIOR, E.K.; GUIMARÃES, V.F. Avaliação da deposição da calda de pulverização em função da vazão e do tipo de bico hidráulico na cultura da soja. Acta Scientiarum. Agronomy, Maringá, v.30, n.2, p.171-175, 2008.

CABEDA, R. Tecnologia de aplicação de herbicidas. In: COOPAVEL/COODETEC/BAYER CropScience (Eds.). Tecnologia de aplicação de defensivos agrícolas II. Cascavel: Bayer CropScience, 2004, p.61-83. (Encontro Técnico, 8)

CHRISTOVAM, R.S.; RAETANO, C.G.; AGUIAR JR., H.O.; DAL-POGETTO, M.H.F.A.; PRADO, E.P.; GIMENES, M.J.; KUNZ, V.L. Assistência de ar em barra de pulverização no controle da ferrugem asiática da soja. Bragantia, Campinas, v.69, n.1, p.231-238, 2010a.

CHRISTOVAM, R.S.; RAETANO, C.G.; PRADO, E.P.; DAL-POGETTO, M.H.F.A.; AGUIAR JR., H.O.; GIMENES, M.J.; SERRA, M.E. Air-assistance and low volume application to control of asian rust on soybean crop. Journal of Plant Protection Research, Poznań, v.50, n.3, p.354-359, 2010b.

CUNHA, J.P.A.R.; TEIXEIRA, M.M.; VIEIRA, R.F.; FERNANDES, H.C. Avaliação de pontas de pulverização hidráulicas na aplicação de fungicida em feijoeiro. Ciência Rural, Santa Maria, v.35, n.5, 1.069-1.074, 2005a.

CUNHA, J.P.A.R.; TEIXEIRA, M.M.; VIEIRA, R.F. Deposição e deriva de calda fungicida aplicada em feijoeiro, em função de bico de pulverização e de volume de calda. Revista Brasileira de Engenharia Agrícola e Ambiental, Campina Grande, v.9, n.1, p.133-138, 2005b.

CUNHA, J.P.A.R.; REIS, E.F.; SANTOS R.O. Controle químico da ferrugem asiática da soja em função de ponta de pulverização e de volume de calda. Ciência Rural, Santa Maria, v.36, n.5, p.1.360-1.366, 2006.

CUNHA, J.P.A.R.; MOURA, E.A.C.; SILVA JÚNIOR, J.L.; ZAGO, F.A.; JULIATTI, F.C. Efeito de pontas de pulverização no controle químico da ferrugem da soja. Engenharia Agrícola, Jaboticabal, v.28, n.2, p.283-291, 2008.

CUNHA, J.P.A.R.; PERES, T.C.M. Influência de pontas de pulverização e adjuvante no controle químico da ferrugem asiática da soja. Acta Scientiarum. Agronomy, Maringá, v.32, n.4, p.597-602, 2010.

FARINHA, J.V.; MARTINS, D.; COSTA, N.V.; DOMINGOS, V.D. Deposição da calda de pulverização em cultivares de soja no estádio R1. Ciência Rural, Santa Maria, v.39, n.6, 2009a.

FARINHA, J.V.; MARTINS, D.; RODRIGUES, A.; ALVES-CARDOSO, L. Depósito del caldo de aspersión de distintos tipos de boquillas en dos cultivares de soya en el estadio V3. Agrociencia, Pelotas, v.43, n.5, p.465-473, 2009b.

FERNANDES, A.P.; PARREIRA, R.S.; FERREIRA, M.C.; ROMANI, G.N.Caracterização do perfil de deposição e do diâmetro de gotas e otimização do espaçamento entre bicos na barra de pulverização. Engenharia Agrícola, Jaboticabal, v.27, n.3, p.728-733, 2007. 
GAZZIERO, D.L.P.; MACIEL, C.D.G.; SOUZA, R.T.; VELINI, E.D.; PRETE, C.E.C.;

OLIVEIRA NETO, W. Deposição de glyphosate aplicado para controle de plantas daninhas em soja transgênica. Planta Daninha, Viçosa-MG, v.24, n.1, p.173-181, 2006.

MACIEL, C.D.; VELINI, E.; BERNARDO, R.S. Desempenho de pontas de pulverização em Brachiaria brizantha cv. MG-4 para controle de ninfas de cigarrinhas das pastagens. Engenharia Agrícola, Jaboticabal, v.27, n.1, p.66-74, 2007.

MADALOSSO, M.G. Espaçamento entre linhas e pontas de pulverização no controle de Phakopsora pachyrhizi Sidow. 2007. 83 f. Dissertação (Mestrado em Engenharia Agrícola) - Centro de Ciências Rurais, Universidade Federal de Santa Maria, Santa Maria, 2007.

NASCIMENTO, J.M.; SOUZA, C.M.A.; GAVASSONI, W.L.; BACCHI, L.M.A.; FENGLER, G.W. Controle de ferrugem asiática da soja utilizando-se diferentes pontas de pulverização em Maracaju-MS. Revista Ciências Técnicas Agropecuárias, La Habana, v.18, n.1, p.1-6, 2009.

QUEIROZ, H.S. Sistema alternativo para redução da evaporação e deriva em aplicações de calda de pulverização baseado na utilização de tubo de vórtice. 2009. 62 f. Dissertação (Mestrado em Engenharia Agrícola) - Unidade Universitária de Ciências Exatas e Tecnológicas, Universidade Estadual de Goiás, Goiânia, 2009.

RAMOS, H.H.; PIO, L.C. Tecnologia de aplicação de produtos fitossanitários. ZAMBOLIM, L.; CONCEIÇÃO, M.Z.; SANTIAGO, T. (Eds.), 3.ed. rev. ampl. O que os Engenheiros Agrônomos devem saber para orientar o uso de produtos fitossanitários. Viçosa: UFV, 2008. p.155-224.

RODRIGUES, A.C.P.; FILHO, S.I.B.S.; MARTINS, D.; COSTA, N.V.; ROCHA, D.C.; SOUZA, G.S.F. Avaliação qualitativa e quantitativa na deposição de calda de pulverização em Commelina benghalensis. Planta Daninha, Viçosa-MG, v.28, n.2, p.421-428, 2010.

RODRIGUES-COSTA, A.C.P.; COSTA, N.V.; CARDOSO, L.A.; PEREIRA, M.R.R.; MARTINS, D. Avaliação de pontas de jato plano na deposição da calda de pulverização com diferentes combinações de plantas de feijão, Brachiaria plantaginea e Bidens pilosa. Planta Daninha, Viçosa, v.28, p.1.159-1.171, 2010. Número especial.

ROMÁN, R.A.A.; CORTEZ, J.W.; FERREIRA, M.C.; OLIVEIRA, J.R.G. Cobertura da cultura da soja pela calda fungicida em função de pontas de pulverização e volumes de calda. Scientia Agraria, Curitiba, v.10, n.3, p.223-232, 2009.

SCUDELER, F.; BAUER, F.C.; RAETANO, C.G. Ângulo de barra e ponta de pulverização na deposição da pulverização em soja. In: SIMPÓSIO INTERNACIONAL DE TECNOLOGIA DE APLICAÇÃO DE AGROTÓXICOS, 3., 2004, Botucatu. Anais... Botucatu: UNESP, 2004. p.13-16.

SOUZA, R.T.; VELINI, E.D.; PALLADINI, L.A. Aspectos metodológicos para análise de depósitos de pulverização pela determinação dos depósitos pontuais. Planta Daninha, Viçosa-MG, v.25, n.1, p.195-202, 2007a.

SOUZA, R.T.; CASTRO, R.D.; PALLADINI, L.A. Depósito de pulverização com diferentes padrões de gotas em aplicações na cultura do algodoeiro. Engenharia Agrícola, Jaboticabal, v.27, n.esp., p.75-82, 2007b.

VELINI, E.D. Estudos e desenvolvimento de métodos experimentais e amostrais adaptados à matologia. 1995. 250 f. Tese (Doutorado em Agronomia) - Faculdade de Ciências Agrárias e Veterinárias, Universidade Estadual Paulista, Jaboticabal, 1995.

VELINI, E.D. Métodos Experimentais. RAETANO, C.A.; ANTUNIASSI, U.R. In: Qualidade em tecnologia de aplicação. Botucatu: FAPEF, 2004. p.54-64.

VIANA, R.G.; FERREIRA, L.R.; TEIXEIRA, M.M.; CECON, P.R.; SOUZA, G.V.R. Avaliação de pontas de pulverização sob diferentes condições operacionais. Engenharia na Agricultura, Viçosa-MG, v.16, n.4, p.428-434/435, 2008. 